Case Report

\title{
Radiofrequency-Targeted Vertebral Augmentation: Case Report of a Patient with 7 Osteoporotic Vertebral Fractures in a Variant of Osteogenesis Imperfecta
}

\author{
Leonard Westermann, Peer Eysel, Marvin Simons, and Kourosh Zarghooni \\ Center for Orthopedic and Trauma Surgery, University Medical Center, Kerpener Str. 62, 50937 Cologne, Germany \\ Correspondence should be addressed to Leonard Westermann; leonard.westermann@uk-koeln.de
}

Received 19 July 2017; Accepted 27 August 2017; Published 4 October 2017

Academic Editor: Koichi Sairyo

Copyright (C) 2017 Leonard Westermann et al. This is an open access article distributed under the Creative Commons Attribution License, which permits unrestricted use, distribution, and reproduction in any medium, provided the original work is properly cited.

\begin{abstract}
Introduction. Radiofrequency-targeted vertebral augmentation (RF-TVA) is a recognized treatment for painful compression fractures. RF-TVA in a patient with multiple compression fractures due to type I osteogenesis imperfecta (OI) has not been previously reported. Case Presentation. A 54-year-old patient with type I OI is presented with a segmental thoracic hyperkyphosis and 7 recent vertebral compression fractures. Because of persistent severe thoracolumbar back pain despite conservative therapy, RF-TVA was indicated. Nocturnal back pain was almost completely relieved at all postoperative time points evaluated. However, overall pain relief dropped only slightly from 7 to 5 on the numerical rating scale (NRS) at the 6-week follow-up, and there was only a small decrease in the Oswestry Disability Index (ODI) from 72\% to 63\%. An MRI at the 3-month follow-up revealed hyperintensity at levels T11 and T12, indicating slight recollapsing. At the 6-month follow-up, the ODI improved to 55\%, although overall pain had worsened to 6 on the NRS. Pain at rest remained at a very low level. Conclusion. Despite the remaining lumbago, RF-TVA may be a good option for patients with OI who have multiple fractures. However, fractures at multiple levels and segmental thoracic hyperkyphosis may increase the risk for recollapsing and ongoing pain.
\end{abstract}

\section{Introduction}

Vertebral compression factures are common and painful in patients with osteoporosis and osteogenesis imperfecta (OI). Adequate treatment is required to relieve serious back pain, restore activities of everyday life, and maintain spine alignment in the sagittal profile. Conservative treatment frequently provides only short-term pain relief $[1,2]$, in which case balloon kyphoplasty is a common procedure when surgery is indicated. Radiofrequency-targeted vertebral augmentation (RF-TVA), developed from the balloon kyphoplasty procedure, is a recent augmentation method that uses high-viscosity cement $[3,4]$. Compared to traditional balloon kyphoplasty, RF-TVA offers a significantly shortened operation time with comparable clinical results and equal, or even reduced, cement leakage rates [5-7]. Recent studies have demonstrated that RF-TVA represents a promising alternative approach in the treatment of compression fractures [8].
We extended the indication of RF-TVA to a 54-yearold woman with multiple compression fractures of Th8-12, L1, and L3, along with diminished osseous stability owing to type I osteogenesis imperfecta and osteoporosis. To our knowledge, this is the first case report describing treatment of a multilevel-fractured spine with RF-TVA.

\section{Case Report}

2.1. History and Examination. A 54-year-old woman with type I OI, osteoporosis ( $T$-Score of -3.3 at the lumbar spine), and at the time untreated Crohn's disease presented at the orthopedic institution with serious thoracolumbar back pain over the previous 5 months and lower back pain over the previous 4 months, without preceding trauma. Several applications of cortisone (in total 3,09g of prednisolone) over the previous 15 years were used for treating her Crohn's disease. 


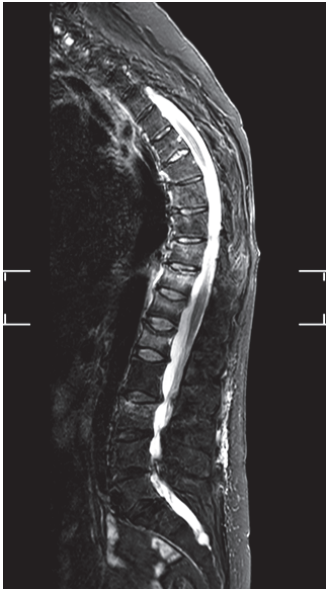

FIGURE 1: Preoperative sagittal magnetic resonance image (STIR sequence) of the lumbar spine showing edema of multiple vertebral bodies.

Because of severe back pain, the patient was not able to walk for more than $500 \mathrm{~m}$ and had several limitations in everyday life. She had experienced approximately 25 fractures without external force since the age of 1 year. Further, she reported having a brother, father, and grandmother, each with osteogenesis imperfecta.

Conservative treatment with pain killers and physiotherapy failed. The recommended bisphosphonate therapy was not started due to time issues. Physical examination revealed hyperkyphosis of the thoracic spine without scoliosis. The patient had throbbing pain of the thoracolumbar spine, and paravertebral myogelosis was observed. There was no sign of spinal cord compression or cauda equine syndrome. The patient rated her back pain as 7/10 on the numerical rating scale (NRS). When lying, the pain worsened to $10 / 10$. The Oswestry Disability Index (ODI) was $72 \%$, constituting a crippling condition. The ODI was determined in German [9].

Radiographs showed multiple compression fractures in the thoracic and lumbar regions, and high signal on the STIR sequence of an MRI was observed at levels Th8-12, L1, and L3 (see also Figure 1). An additional older compression fracture was present at L4 without any enhanced signal. The patient subsequently underwent RF-TVA.

Regarding the sagittal profile, $\mathrm{X}$-ray of the whole vertebrae showed a thoracic hyperkyphosis of 70 degrees and a C7 offset of $4.3 \mathrm{~cm}$.

2.2. Operation. The patient was placed in a prone position on a radiolucent operating table. The compression fractures were localized fluoroscopically using a conventional C-arm device (Endura/Philips/Netherlands). Under fluoroscopic guidance, an introducer was inserted through a small skin incision into either the right or the left pedicle at levels T8-T12 and L3. Access was bipedicular only at level L1. The navigational MidLine Osteotome (DFINE) was inserted through the introducer and guided fluoroscopically. After creating a threedimensional cavity in the center of the fractured vertebrae, the radiofrequency-activated cohesive ultrahigh-viscosity
PMMA cement was delivered at a controlled rate into the cavity under continuous pressure from the hydraulic assembly. The cement volume per vertebral body ranged from 2.2 to $4.3 \mathrm{~mL}$. The intent was to restore and strengthen the vertebrae by injecting the cement primarily into the anterior part of each vertebral body. The total time from incision to suturing was 100 minutes. The patient tolerated the intervention well without pulmonary or neurological complications.

2.3. Postoperative Course. The procedure was conducted during a brief in-hospital stay. Immediately after the treatment, the patient's NRS back pain rating decreased from 7 to 5 , allowing some activities of her daily life to be restored. Pain reduction in the lying position was more evident, with a decrease in the NRS rating from 10 to 2 . Immediately after surgery, the patient reported being able to sleep at night without pain interruptions. At the 6-week follow-up, the ODI had decreased from $72 \%$ to $63 \%$.

Figure 2 shows postoperative radiographs with integrated PMMA at affected sites.

Because of ongoing pain 3 months after surgery, we performed another MRI of the lumbar spine. The MRI revealed small hyperintense signals at levels T11 and T12, indicating the possibility of slight recollapsing (see also Figure 3). At the 6month follow-up, the patient stated that her overall back pain had worsened to a rating of 6 on the NRS, and the patient still was not able to work. However, pain at night remained almost completely relieved. Although the ODI improved to 55\%, this still constituted a severe disability.

\section{Discussion}

The patient in this study had type I osteogenesis imperfecta, which is the most benign form of a group of connective tissue disorders that cause skeletal abnormalities such as bone fragility and deformity [10]. Vertebral compression fractures often occur with age or disease processes due to deteriorating bone consistency [11]. In this case, the patient experienced 7 vertebral compression fractures due to low bone density ( $T$-Score of -3.3) associated with OI and osteoporosis secondary to long-lasting cortisone treatment for Crohn's disease.

Corporal disability and severe lumbago unresponsive to conservative therapy indicated that surgical intervention was appropriate for the patient. Several operative procedures were available for treatment, but the large number of fractures and very low bone quality limited the practical options. Standard approaches would have required extensive instrumentation, with high risks of screw loosening, establishing bone union, and perioperative complications. Under these circumstances, we considered vertebroplasty and kyphoplasty as possible alternatives before selecting RF-TVA. This treatment had several novel characteristics and improvements over standard therapy. In particular, the operating time in RF-TVA was shortened because the unipedicular approach is often sufficient to achieve adequate cement distribution $[3,7,12]$. Simultaneously, there is also a reduction in the risk of spinal cord damage with single transpedicular access. While treatment success with RF-TVA is well documented in the 


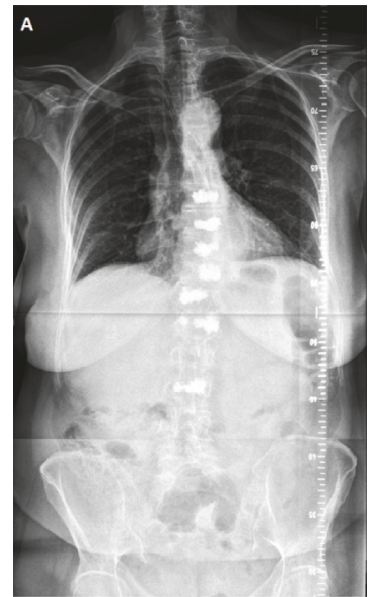

(a)

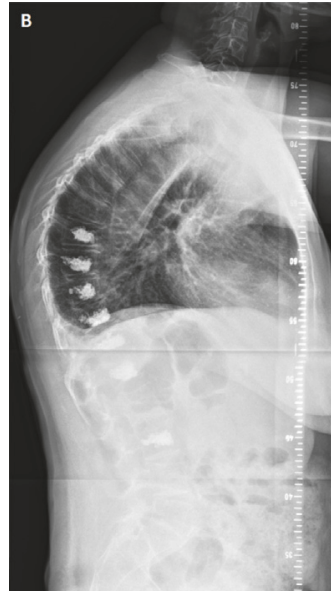

(b)

Figure 2: Postoperative anteroposterior (a) and lateral (b) radiographs of the spine.

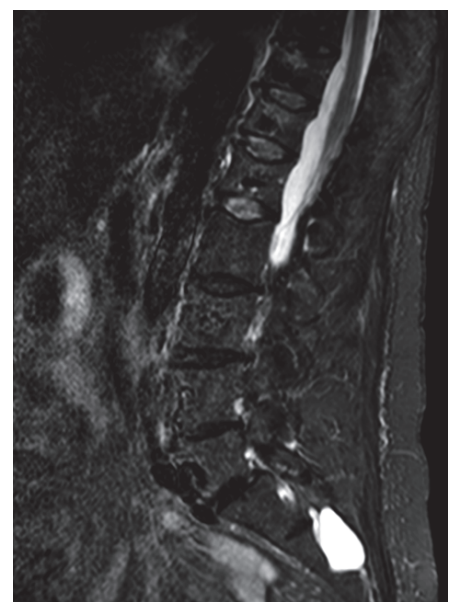

FIGURE 3: Postoperative sagittal magnetic resonance image of the spine 3 months after surgery, showing increased signal and signs of slight recollapsing, especially in the thoracolumbar transition on the STIR sequence.

literature, there are currently no studies that have evaluated RF-TVA for patients with only OI $[6,7,13]$. The few published case reports dealing with OI used conventional vertebroplasty or kyphoplasty $[14,15]$.

Similar to balloon kyphoplasty, RF-TVA is associated with some common adverse events. However, both procedures are considered safe and the rates of PMMA extravasation are low $[6,7]$. It is possible that published complications in OI cases may be related to increased bone porosity in these patients $[16,17]$. We expected that RF-TVA would have an advantage relative to balloon kyphoplasty in minimizing the destruction of remaining native spongiosa structures during balloon expansion [18]. This relatively gentle approach could be even more important in similar cases with both OI and severe osteoporosis. It may be possible to preserve more intact cancellous bone while using a navigational osteotome to create a desired transpedicular path via only a single pedicle.
Although the patient reported noticeable pain relief, she remained disabled because of ongoing severe lumbago and was consequently not able to work. Nevertheless, the patient appreciated the pain relief, which enabled her to sleep continuously and improved her quality of life. Her stagnating improvement at the 6-month follow-up may have been related to the disease severity and the presence of fractures across 7 vertebral levels. Most published studies have evaluated patients with only up to 3-level fractures. The patient did not have an adjacent segment fracture, though she had a segmental thoracic hyperkyphosis, which might be a risk factor for new fractures at adjacent levels [19]. Adjacent fractures are a frequent complication after kyphoplasty with an incidence rate of 3-29\% [20].

Another reason for ongoing pain may have been the application of an insufficient cement volume in some of the vertebrae. This could have resulted in the enhanced signals seen on the postoperative magnetic resonance image 3 months after surgery, indicating a process of recollapsing without significant secondary loss of height restoration. There is no recommendation in the literature for a specific amount of cement to achieve optimal results, although a biomechanical study by Liebschner et al. suggests that $3.5 \mathrm{~mL}$ in $\mathrm{L} 1$ is necessary to adequately restore stiffness [21]. However, other authors suggest that larger volumes are necessary [22, 23]. Clinical data show that smaller volumes of cement $(2.9 \mathrm{~mL}$ in thoracic vertebrae and $3.0 \mathrm{~mL}$ in lumbar vertebrae) may be sufficient to reduce pain and restore vertebral height [24]. A recent review by Papanastassiou et al. reported that there is growing evidence of a correlation between larger cement volumes and both better restoration of sagittal alignment and improved pain resolution [24-28]. The mean volume of cement used in the different studies (ranging from 3.4 to $4.0 \mathrm{~mL}$ ) was probably slightly higher in comparison to this study [6].

RT-TVA should be considered as a potential treatment for patients with OI who have numerous vertebral fractures and for whom conservative treatment has been ineffective. We believe that it is probably the most suitable surgical 
procedure in such cases, even though the pain relief observed in this study was not comparable to what may be expected for patients with 1-level or 2-level fractures. The patient reported that the initial pain relief at night immediately after RF-TVA was of sufficient value that in hindsight she would elect to undergo the treatment again.

\section{Conclusion}

RF-TVA is a minimally invasive therapeutic procedure offering an alternative to standard treatments for multiple vertebral fractures in patients with type I osteogenesis imperfecta and pain that is unresponsive to conservative treatment. In this case report, the patient improved only slightly in overall ODI and pain level, but this may have been because of the multilevel fractures or segmental kyphosis. The patient did, however, experience immediate and significant pain relief at night, which led her to report that the procedure was worthwhile.

\section{Consent}

Written informed consent for publication of the clinical details and/or clinical images was obtained from the patient.

\section{Disclosure}

This manuscript was written in accordance with the current CARE guidelines for case reports [29].

\section{Conflicts of Interest}

Each author certifies that he or she has no commercial associations (e.g., consultancies, stock ownership, equity interest, or patent/licensing arrangements) that might pose conflicts of interest in connection with the submitted article.

\section{Authors' Contributions}

Leonard Westermann made substantial contributions to conception and design, acquisition of data, and analysis and interpretation of data and participated in drafting and writing the article. Peer Eysel made substantial contributions to analysis and interpretation of data and participated in revising it critically for important intellectual content. Marvin Simons contributed to acquisition and interpretation of data and participated in drafting and writing the article. Kourosh Zarghooni made substantial contributions to conception and design and participated in revising the paper critically for important intellectual content. All authors read and approved the final manuscript.

\section{References}

[1] G. P. Lyritis, B. Mayasis, N. Tsakalakos et al., "The natural history of the osteoporotic vertebral fracture," Clinical Rheumatology, vol. 8, no. 2, pp. 66-69, 1989.

[2] C. L. Goldstein, N. B. Chutkan, T. J. Choma, and R. D. Orr, "Management of the elderly with vertebral compression fractures," Neurosurgery, vol. 77, no. 4, pp. S33-S45, 2015.
[3] A. A. Kurth, H. Bayer-Helms, C. Böwe et al., "Radiofrequency kyphoplasty - a novel vertebral augmentation system: a prospective, multi-center observational study," Osteologie, vol. 21, no. 3, pp. 174-179, 2012.

[4] R. Bornemann, K. Kabir, L. A. Otten et al., "Radiofrequency Kyphoplasty - An Innovative method for the treatment of vertebral compression fractures - Comparison with conservative treatment," Zeitschrift fur Orthopadie und Unfallchirurgie, vol. 150, no. 4, pp. 392-396, 2012.

[5] S. G. Mattyasovszky, A. A. Kurth, P. Drees, J. Gemidji, S. Thomczyk, and K. Kafchitsas, "Minimally invasive cement augmentation of osteoporotic vertebral compression fractures with the new radiofrequency kyphoplasty," Operative Orthopadie und Traumatologie, vol. 26, no. 5, pp. 497-512, 2014.

[6] H.-J. Riesner, K. Kiupel, P. Lang, F. Stuby, B. Friemert, and H.G. Palm, "Clinical relevance of cement leakage after radiofrequency kyphoplasty vsballoon kyphoplasty: a prospective randomised study," Zeitschrift fur Orthopadie und Unfallchirurgie, vol. 154, no. 4, pp. 370-376, 2016.

[7] A. Petersen, E. Hartwig, E. M. W. Koch, and M. Wollny, "Clinical comparison of postoperative results of balloon kyphoplasty (BKP) versus radiofrequency-targeted vertebral augmentation (RF-TVA): a prospective clinical study," European Journal of Orthopaedic Surgery and Traumatology, vol. 26, no. 1, pp. 67-75, 2016.

[8] L. Oberkircher, J. Struewer, C. Bliemel et al., "Height restoration and preservation in osteoporotic vertebral compression fractures: a biomechanical analysis of standard balloon kyphoplasty versus radiofrequency kyphoplasty in a cadaveric model," Journal of Spinal Disorders and Techniques, vol. 27, no. 5, pp. 283289, 2014.

[9] A. F. Mannion, A. Junge, J. C. T. Fairbank, J. Dvorak, and D. Grob, "Development of a german version of the oswestry disability index. part 1: cross-cultural adaptation, reliability, and validity," European Spine Journal, vol. 15, no. 1, pp. 55-65, 2006.

[10] A. Forlino and J. C. Marini, "Osteogenesis imperfecta," The Lancet, vol. 387, no. 10028, pp. 1657-1671, 2016.

[11] C. Dewar, "Diagnosis and treatment of vertebral compression fractures," Radiol Technol, vol. 86, no. 3, pp. 301-320, 2015.

[12] F. A. Elgeti, T. Marnitz, T. J. Kröncke, and B. Gebauer, "DFine radiofrequency kyphoplasty (RFK) - kyphoplasty with ultrahigh viscosity cement," RoFo Fortschritte auf dem Gebiet der Rontgenstrahlen und der Bildgebenden Verfahren, vol. 182, no. 9, pp. 803-805, 2010.

[13] R. Bornemann, M. Hanna, K. Kabir, H. Goost, D. C. Wirtz, and R. Pflugmacher, "Continuing conservative care versus crossover to radiofrequency kyphoplasty: a comparative effectiveness study on the treatment of vertebral body fractures," European Spine Journal, vol. 21, no. 5, pp. 930-936, 2012.

[14] P. M. Rami, J. K. McGraw, E. V. Heatwole, and J. M. Boorstein, "Percutaneous vertebroplasty in the treatment of vertebral body compression fracture secondary to osteogenesis imperfecta," Skeletal Radiology, vol. 31, no. 3, pp. 162-165, 2002.

[15] C. H. Fürstenberg, T. Grieser, B. Wiedenhöfer, H. J. Gerner, and C. M. Putz, "The role of Kyphoplasty in the management of osteogenesis imperfecta: risk or benefit?" European Spine Journal, vol. 19, no. 2, pp. S144-S148, 2010.

[16] P. Tozzi, Y. Abdelmoumene, A. F. Corno, P. A. Gersbach, H.-M. Hoogewoud, and L. K. von Segesser, "Management of pulmonary embolism during acrylic vertebroplasty," Annals of Thoracic Surgery, vol. 74, no. 5, pp. 1706-1708, 2002. 
[17] C. Vasconcelos, P. Gailloud, J.-B. Martin, and K. J. Murphy, "Transient arterial hypotension induced by polymethylmethacrylate injection during percutaneous vertebroplasty," Journal of Vascular and Interventional Radiology, vol. 12, no. 8, pp. 1001-1002, 2001.

[18] R. Bornemann, E. M. W. Koch, M. Wollny, and R. Pflugmacher, "Treatment options for vertebral fractures an overview of different philosophies and techniques for vertebral augmentation," European Journal of Orthopaedic Surgery and Traumatology, vol. 24, no. 1, pp. S131-S143, 2014.

[19] P. A. Hulme, J. Krebs, S. J. Ferguson, and U. Berlemann, "Vertebroplasty and kyphoplasty: a systematic review of 69 clinical studies," Spine, vol. 31, no. 17, pp. 1983-2001, 2006.

[20] D. Fribourg, C. Tang, P. Sra, R. Delamarter, and H. Bae, "Incidence of subsequent vertebral fracture after kyphoplasty," Spine, vol. 29, no. 20, pp. 2270-2276, 2004.

[21] M. A. K. Liebschner, W. S. Rosenberg, and T. M. Keaveny, "Effects of bone cement volume and distribution on vertebral stiffness after vertebroplasty," Spine, vol. 26, no. 14, pp. 15471554, 2001.

[22] S. Molloy, J. M. Mathis, and S. M. Belkoff, "The effect of vertebral body percentage fill on mechanical behavior during percutaneous vertebroplasty," Spine, vol. 28, no. 14, pp. 15491554, 2003.

[23] S. M. Belkoff, J. M. Mathis, L. E. Jasper, and H. Deramond, "The biomechanics of vertebroplasty: The effect of cement volume on mechanical behavior," Spine, vol. 26, no. 14, pp. 1537-1541, 2001.

[24] M. Röllinghoff, A. Hagel, J. Siewe et al., "Is height restoration possible with a comparatively smaller amount of cement in radiofrequency kyphoplasty using a monopedicle approach?" Zeitschrift fur Orthopadie und Unfallchirurgie, vol. 151, no. 2, pp. 156-162, 2013.

[25] A. Krüger, G. Baroud, D. Noriega et al., "Height restoration and maintenance after treating unstable osteoporotic vertebral compression fractures by cement augmentation is dependent on the cement volume used," Clinical Biomechanics, vol. 28, no. 7, pp. 725-730, 2013.

[26] C. Xu, H.-X. Liu, and H.-Z. Xu, "Analysis of related factors on the deformity correction of balloon kyphoplasty," American Journal of Neuroradiology, vol. 35, no. 1, pp. 202-206, 2014.

[27] C. Röder, B. Boszczyk, G. Perler, E. Aghayev, F. Külling, and G. Maestretti, "Cement volume is the most important modifiable predictor for pain relief in BKP: Results from SWISSspine, a nationwide registry," European Spine Journal, vol. 22, no. 10, pp. 2241-2248, 2013.

[28] I. D. Papanastassiou, A. Filis, M. A. Gerochristou, and F. D. Vrionis, "Controversial issues in kyphoplasty and vertebroplasty in osteoporotic vertebral fractures," BioMed Research International, vol. 2014, Article ID 934206, 12 pages, 2014.

[29] D. S. Riley, M. S. Barber, G. S. Kienle et al., "Care guidelines for case reports: explanation and elaboration document," Journal of Clinical Epidemiology, 2017. 


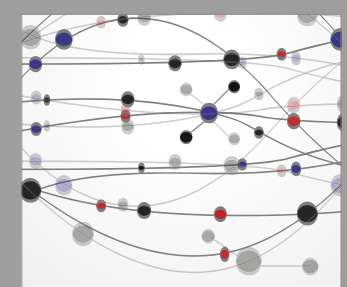

The Scientific World Journal
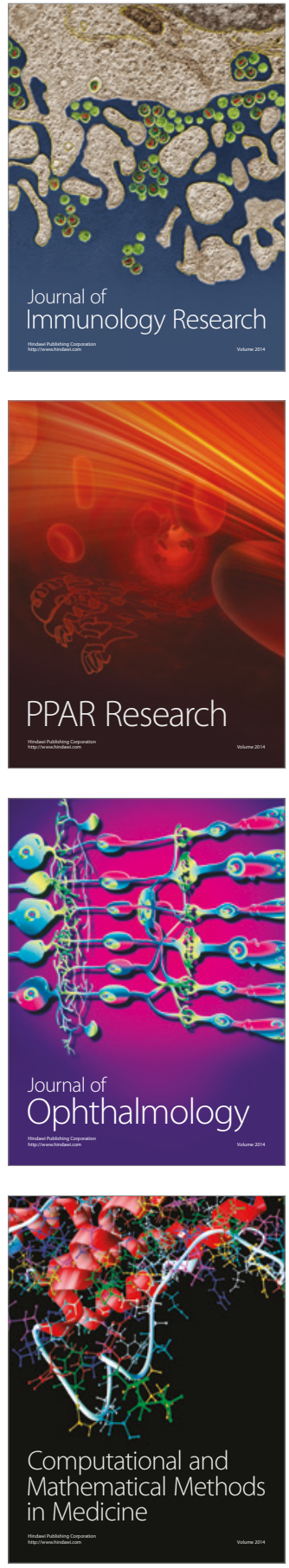

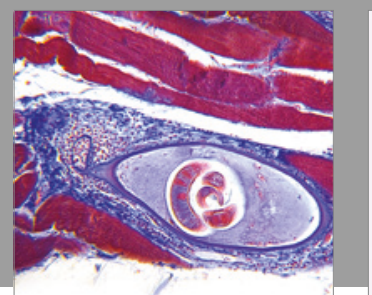

Gastroenterology Research and Practice
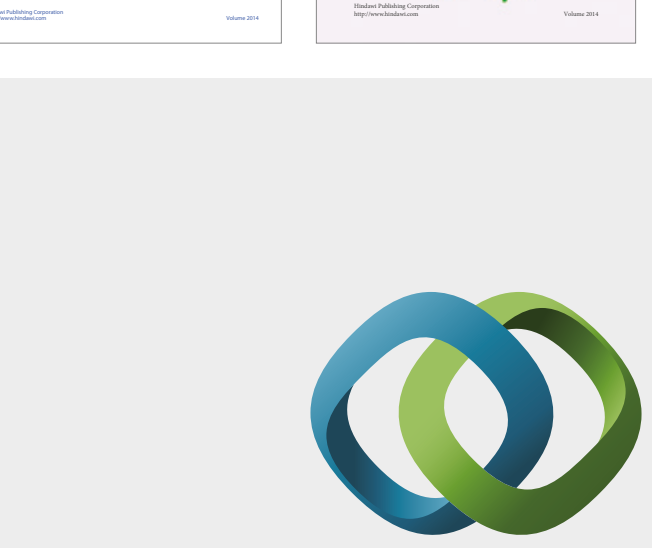

\section{Hindawi}

Submit your manuscripts at

https://www.hindawi.com
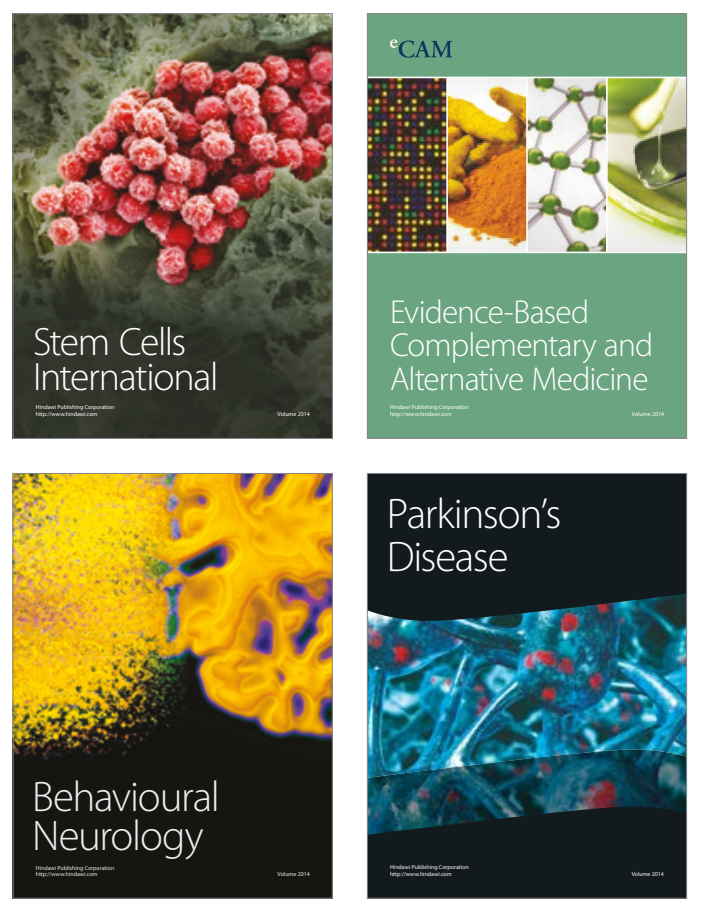
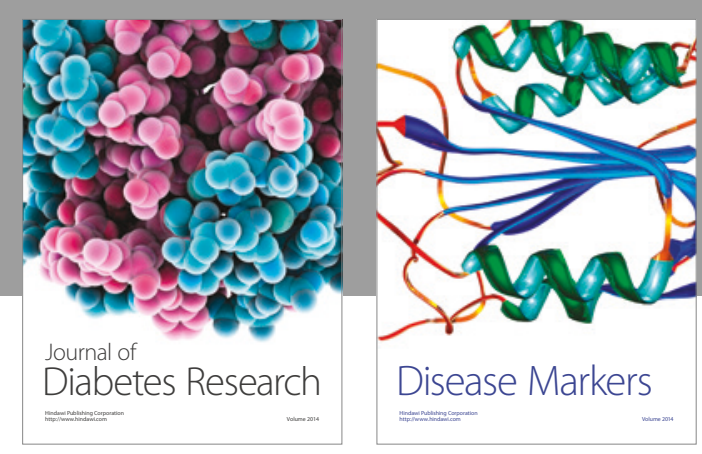

Disease Markers
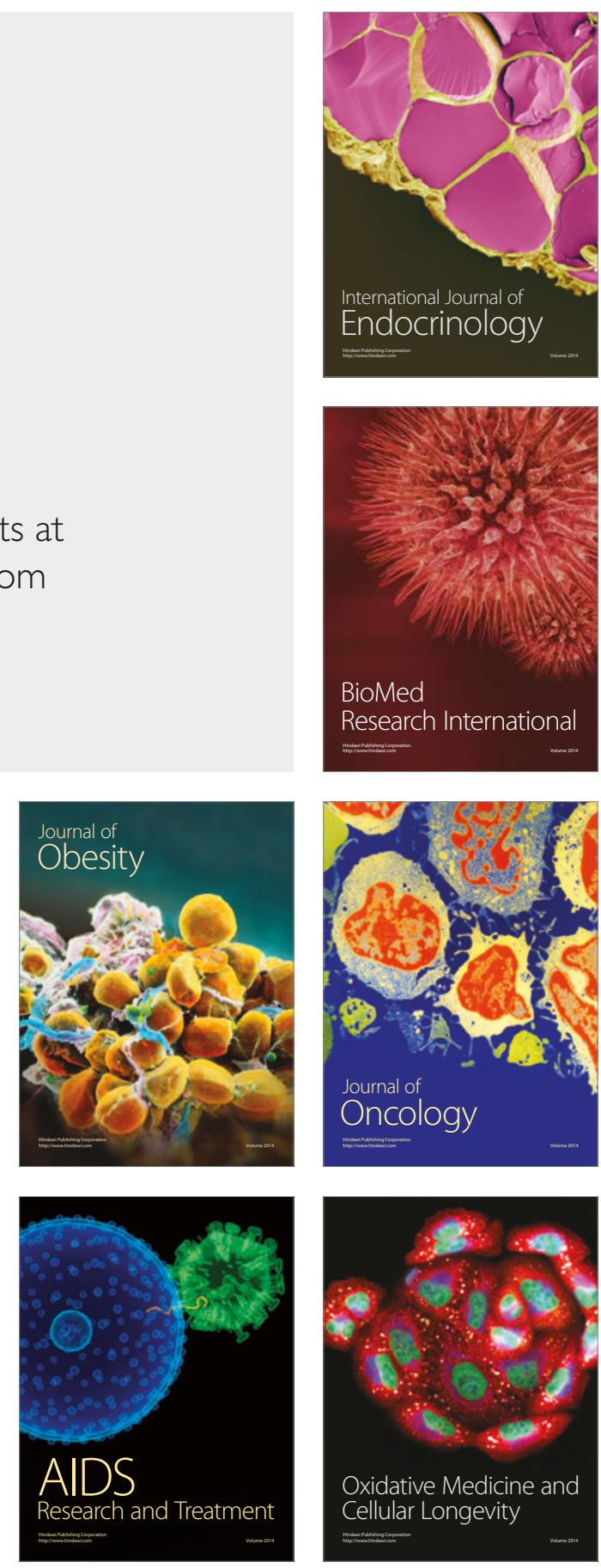